\title{
Aprendizagem em cursos de inclusão digital para pessoas adultas e idosas
}

\author{
Johannes Doll", Caroline Stumpf Buaes*
}

\section{Resumo}

A inclusão digital no Brasil está avançando, porém de forma desigual em relação às faixas etárias: uma grande parte dos adultos e idosos não sabe usar um computador e existem poucas iniciativas para incluir este grupo dentre os usuários dos recursos da informática. Para compreender melhor este desafio, o presente estudo analisa as aprendizagens de pessoas com mais de 45 anos durante cursos de introdução ao uso do computador voltados para esta faixa etária, por meio de um instrumento de observação do desempenho em várias habilidades. Os resultados apontam para ritmos diferentes de aprendizagem, bem como para certa influência da idade na execução de em algumas habilidades. A discussão dos dados apresentada neste estudo está embasada em teorias psicológicas sobre a aprendizagem.

Palavras-chave: Inclusão digital. Aprendizagem. Envelhecimento.

\section{Introdução}

Observa-se que a inclusão digital no Brasil está apresentando significativos avanços. Os dados da Pesquisa Nacional por Amostragem de Domicílio (PNAD) apontaram, em 2001, que um grupo ainda muito pequeno teve acesso a um computador $(12,5 \%)$ e menor ainda à internet (8,3\%). Somente cinco anos mais tarde, esses números quase duplicaram. A PNAD 2006 mostra que neste ano $22,2 \%$ da população brasileira tiveram acesso a um computador em casa e $16,8 \%$ possuíram internet. Também fora de casa o uso do internet aumentou. Segundo os dados do PNAD 2005, 21\% dos brasileiros declararam que usaram a internet, pelo menos, uma vez durante os últimos três meses (Tab. 1).

Uma análise mais detalhada demonstra, porém, enormes diferenças, dependendo da escolaridade, da renda $\mathrm{e}$ da idade, bem como grandes diferenças regionais. Em relação às diferentes faixas

* Doutorado em Educação pela Universitat Koblenz Landau (Alemanha). Professor da Faculdade de Educação da Universidade Federal do Rio Grande do Sul e do Programa de Pós-Graduação em Educação da Universidade Federal do Rio Grande do Sul. Endereço para correspondência: Universidade Federal do Rio Grande do Sul, Faculdade de Educação - Departamento de Ensino e Currículo. Rua Paulo Gama, s/nº, prédio 12201, sala 908, bairro Farroupilha. CEP 90046-900, Porto Alegre - RS. E-mail: johannes.doll@ufrgs.br.

** Mestra em Educação pela Universidade Federal do Rio Grande do Sul. Professora do curso de Psicologia do Centro Universitário Franciscano.

$\hookrightarrow$ Recebido em agosto de 2009 - Avaliado em março de 2010.

$\rightarrow$ doi:10.5335/rbceh.2009.031 
etárias, são principalmente os jovens que usam a internet.

Tabela 1 - Uso do internet segundo grupos de idade.

\begin{tabular}{cc}
\hline Faixa etária & $\begin{array}{c}\text { Uso do internet nos últimos } \\
\text { três meses }\end{array}$ \\
\hline De 10 a 19 anos & $31,6 \%$ \\
De 20 a 29 anos & $29,9 \%$ \\
De 30 a 39 anos & $17,9 \%$ \\
De 40 a 49 anos & $12,7 \%$ \\
De 50 a 59 anos & $6,1 \%$ \\
60 anos e mais & $1,8 \%$ \\
\hline
\end{tabular}

Fonte: PNAD 2005.

Os dados sobre inclusão digital da pesquisa "Idosos no Brasil", realizada em 2006 pela Fundação Perseu Abramo e Sesc/SPA com 3.759 pessoas, confirmam as tendências apontadas, demonstrando que o uso do computador, ou até o contato com o mesmo, está fortemente vinculado à idade do usuário (Fig. 1), à formação escolar e à renda familiar. Entre os idosos, $88 \%$ conheciam um computador de vista, mas somente $8 \%$ já o tinham usado. Entre as pessoas que nunca frequentaram a escola, somente $68 \%$ já tinham visto um computador e somente $1 \%$ já o tinha utilizado. (DOLL, 2007).

A pouca participação de pessoas com mais idade no mundo da informática também é confirmada em outros países. $\mathrm{Na}$ Alemanha, por exemplo, somente um pequeno e específico grupo de idosos se interessa pela internet. Estes são principalmente homens, idosos mais jovens, com boa formação escolar. Nos outros grupos de idosos, apesar de existirem diferentes incentivos que promovam a inclusão digital, o número de internautas idosos - chamados Silver Surfer - não está aumentando. Em alguns grupos até está diminuindo: pessoas chamadas de Off-liner assumem sua resistência contra a entrada no mundo virtual. (DOH). Resultados semelhantes são encontrados em outros países, como nos Estados Unidos. (US DEPARTMENT..., 2000). Selwyn et al. (2003) apontam no seu estudo sobre a inclusão digital na Inglaterra, contrariando o discurso forte de inclusão digital promovido por determinados grupos, que existem muitas pessoas idosas que não utilizam o computador por opção, não por impossibilidade de acessar.

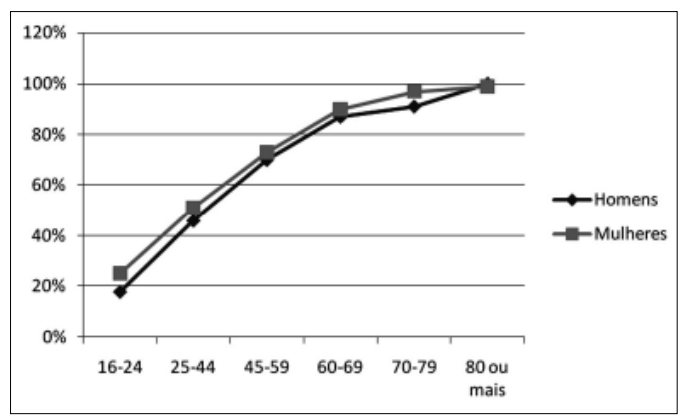

Fonte: Pesquisa "Idosos no Brasil”, Fundação Perseu Abramo, SESC/SP, 2007.

Figura 1 - Distribuição das pessoas que nunca utilizaram um computador, por faixa etária e sexo.

Ao contrário de ações de inclusão digital de pessoas adultas maduras e idosas na Europa e nos Estados Unidos, encontramos ainda poucas iniciativas no Brasil. A maioria se vincula às universidades da terceira idade (KACHAR, 2003), além de iniciativas isoladas de algumas prefeituras. Se é para a escola que se destinam recursos para o acesso à aprendizagem e ao uso desses equipamentos, como ficam aqueles/as que deixaram a escola muitos anos atrás? 
Pensando na sociedade que possui cada vez mais sujeitos adultos maduros (maiores de 45 anos) e idosos que sequer têm uma maior proximidade com a escola, como incluí-los nesse processo de aprendizagem digital?

Essas questões contribuíram para a organização de um projeto de pesquisa e extensão na universidade " $X$ " voltado a oferecer aos maiores de 45 anos um aprendizado básico de informática. No contexto brasileiro, percebe-se uma maior produção científica acerca dos processos de aprendizagem de crianças e pouca tradição de estudos sobre esse processo em adultos e idosos. As reflexões teóricas sobre práticas e experiências educacionais com adultos maiores e seus processos de aprendizagem são escassas. No que tange à educação de adultos, a pedagogia desenvolveu-se mais no campo da alfabetização e da educação de jovens e adultos. (DOLL, 2002).

Nesse sentido, além de oferecer um curso de informática básico, a equipe executora tem uma preocupação com os processos de aprendizagem do públicoalvo composto de alunos mais velhos. As questões que guiam as ações educativas de pesquisa são: $O$ que os alunos aprendem? Como avaliar o que os alunos aprendem? Neste estudo, selecionamos seis habilidades básicas para o uso do computador que foram avaliadas por meio de observação e de preenchimento de fichas de indicadores de aprendizagem.

\section{Contexto dos cursos e perfil dos participantes}

Foram realizados três cursos de introdução ao uso do computador em
2006. Cada um teve a duração de 20 horas, durante quatro semanas, com dois encontros semanais no turno da noite, sendo as aulas realizadas nas dependências do laboratório de informática "Y/X". As turmas eram compostas por, no máximo, 15 alunos, uma professora e monitores, que realizavam tanto as observações e preenchimento das fichas de avaliação como prestavam auxílio aos alunos, assumindo, assim, a função de mediadores.

O público-alvo eram sujeitos adultos com mais de 45 anos que foram captados por meio de anúncios do curso em jornais (Z; W). A taxa de matrícula era de baixo valor e a inscrição foi realizada por ordem de chegada, em virtude da grande repercussão nos meios de divulgação. Os alunos foram entrevistados, responderam a questionários e sabiam que estavam sendo observados por se tratar de uma atividade de pesquisa.

Participaram do estudo 37 sujeitos com idades entre 47 e 76 anos. No grupo havia 24 mulheres e 13 homens. A distribuição da escolaridade mostrou que dois sujeitos possuíam ensino fundamental incompleto; quatro, ensino fundamental completo; quatro, ensino médio incompleto; oito, ensino médio completo; cinco, ensino superior incompleto e 14, ensino superior completo. Em relação às experiências prévias, 26 possuem computador em casa, contudo apenas 18 sujeitos já haviam tido contato com este recurso. Tal fato pode indicar o uso do computador por filhos e netos e a vontade de iniciar-se no campo da informática, utilizando os diferentes recursos que os programas computacionais e a internet podem oferecer. 


\section{Aspectos metodológicos}

No contexto dos nossos cursos de inclusão digital para pessoas adultas maduras e idosas, ao longo das pesquisas realizadas desde o ano de 2002, experimentamos diferentes formas de coleta de informações acerca das aprendizagens dos alunos, que apresentaram alguns problemas, dos quais se destacam: tópicos demais a serem observados, dificuldade na classificação da observação, interferências no andamento das aulas, etc.

Refletindo sobre as experiências dos cursos anteriores, construímos uma ficha de observação que se concentra em dez tópicos. Quatro tópicos referem-se a estratégias de aprendizagem, à compreensão das tarefas, à execução das tarefas em aula e à autonomia do aluno; os outros seis aspectos referem-se às habilidades consideradas importantes para usar o computador: habilidade com o mouse, interação com a tela encontrando os ícones, abrir um programa, fechar o programa, desenvoltura em digitar, vínculo teclado/mouse - tela. É claro que existe o perigo de resultados deste tipo de investigação serem influenciados pela subjetividade do observador. Contudo, além da descrição precisa do comportamento desejado nas fichas de observação, foi realizado um treinamento com os observadores que acompanharam o curso inteiro, o que torna os dados mais confiáveis. Outra medida para tornar os resultados mais confiáveis foi manter em todos os cursos os mesmos observadores.

No presente estudo utilizamos dados coletados em três cursos realizados em 2006 referentes às seis habilidades citadas para discutir as aprendizagens dos participantes. A avaliação dos indicadores de aprendizagem foi realizada por meio do preenchimento da ficha de observação pelos monitores em três momentos distintos: início, meio e fim dos cursos. A observação foi realizada por meio de lista das habilidades que deveriam ser classificadas em cinco níveis conforme o desempenho do aluno (escala de Likert). A partir dela produziu-se uma escala de intervalos, condição exigida para a realização do teste $t$ de Student e correlação de Pearson.

Antes da discussão dos resultados, é importante ainda refletir sobre a abrangência e os limites do estudo. Em primeiro lugar, trata-se de um grupo relativamente pequeno de 37 participantes, que representam uma seleção positiva, já que tiveram a iniciativa de se inscrever num curso de computação. Diante do grande número de adultos maduros e idosos que possuem pouco contato e demonstram pouco interesse pelo computador, como referido na introdução, os participantes certamente não são representativos para esta faixa etária, um problema, que, aliás, existe também em outros estudos sobre a inclusão digital de adultos e idosos. (SELWYN et al., 2003).

A segunda restrição deve-se ao instrumento de observação, que indica mudanças observáveis de comportamento dos participantes, porém não esclarece, necessariamente, as razões de uma mudança mais rápida ou lenta. Tendo em vista que o processo de aprendizagem, especialmente de pessoas adultas e idosas, pode ser influenciado por diferentes 
fatores, cabe a outros estudos especificar o peso desses no processo de aprendizagem de pessoas deste segmento etário.

\section{Apresentação e discussão dos dados}

As duas questões que orientaram este estudo sobre a avaliação da aprendizagem das pessoas adultas e idosas participantes dos cursos de inclusão digital foram: Os dados mostram, de fato, um progresso de aprendizagem? Há relação da aprendizagem com a idade dos sujeitos?

A aprendizagem das seis habilidades consideradas importantes para o uso do computador - habilidade com o mouse, interação com a tela encontrando os ícones, abrir um programa, fechar o programa, destreza em digitar e vínculo teclado/mouse/tela - foram avaliadas pelo cálculo da média do desempenho em cada habilidade e comparando com as médias do início e do final do curso. Utilizou-se como instrumento de análise dos dados o teste $t$ de Student com o objetivo de comparar a média de desempenho das tarefas (seis habilidades) nos três momentos da avaliação. Os dados foram analisados com o auxílio do software SPSS. Interpretamos como comprovação de aprendizagens os resultados do teste que apontaram uma diferença significativa entre os diferentes momentos. A Tabela 2 apresenta a distribuição da média de desempenho das habilidades.

Tabela 2 - Desempenho nas habilidades.

\begin{tabular}{lcccccc}
\hline \multicolumn{1}{c}{ Habilidade } & $\begin{array}{c}\text { Média no } \\
\text { início }\end{array}$ & $\begin{array}{c}\text { Média na } \\
\text { metade }\end{array}$ & $\begin{array}{c}\text { Média } \\
\text { no final }\end{array}$ & $\begin{array}{c}\text { Diferença início } \\
\text { e metade }\end{array}$ & $\begin{array}{c}\text { Diferença } \\
\text { metade e final }\end{array}$ & $\begin{array}{c}\text { Diferença } \\
\text { início e final }\end{array}$ \\
\hline Lidar com mouse & 2,14 & 1,46 & 1,30 & $0,68^{\star}$ & 0,16 & $0,84^{\star \star}$ \\
Orientação na tela & 2,11 & 1,75 & 1,32 & 0,36 & $0,43^{\star}$ & $0,79^{\star \star}$ \\
Abrir o programa & 2,11 & 1,69 & 1,03 & 0,42 & $0,66^{\star \star}$ & $1,08^{\star \star}$ \\
Fechar o programa & 2,19 & 1,75 & 1,05 & 0,44 & $0,70^{\star \star}$ & $1,14^{\star \star}$ \\
Digitar & 2,24 & 1,89 & 1,41 & 0,35 & $0,48^{*}$ & $0,83^{\star \star}$ \\
Relação tela e mouse & 1,86 & 1,64 & 1,24 & 0,22 & $0,40^{*}$ & $0,62^{\star}$ \\
\hline
\end{tabular}

* Diferença significativa para um nível de significância de 0,01 .

* Diferença significativa para um nível de significância de 0,05.

Ao comparar os dados do início e do final do curso, percebe-se uma diferença altamente significativa (1\%) em todas as habilidades, com exceção da relação tela/mouse, em que a diferença é significativa em nível de 5\%. Dessa forma, os resultados permitem afirmar que houve avanço no desenvolvimento em todas as habilidades.

A análise das diferenças entre início, metade e final do curso permite indicar o momento em que se deram as mudanças mais significativas, isto é, as aprendizagens. Todas as habilidades tiveram um maior desenvolvimento a partir da metade do curso, com exceção da relação com o mouse, cujo avanço aconteceu na primeira etapa. Assim, podemos apontar que certas habilidades precisam de mais tempo, treino e exercício para serem assimiladas. 
Essas habilidades, além de terem sido exercitadas durante o curso, foram experimentadas pelos sujeitos que possuíam computador em suas casas, já que a maior parte do grupo compartilhava dessa situação. Nesse sentido, Nabas e Xavier (2004) apontam que o treinamento repetitivo alivia a carga atencional em decorrência da automação. Enquanto as operações isoladas não se automatizam, a execução de cada uma delas constitui o objetivo de certa parte da atividade e atrai atenção para si. (LURIA, 1991). Então, com o treinamento ao longo das aulas, o desempenho lento, serial e mediado verbalmente foi sendo substituído pelo desempenho rápido, requerendo menos esforços voluntários.

Para examinar a existência de uma relação entre a idade e as habilidades básicas avaliadas no curso foi realizada uma análise de correlação de Pearson. De forma geral, os resultados indicam uma correlação muito fraca entre a idade e a habilidade de lidar com os desafios de um computador em nível de iniciantes. As idades dos participantes variavam entre 47 e 76 anos. A Figura 2 apresenta a distribuição dos participantes por idade.

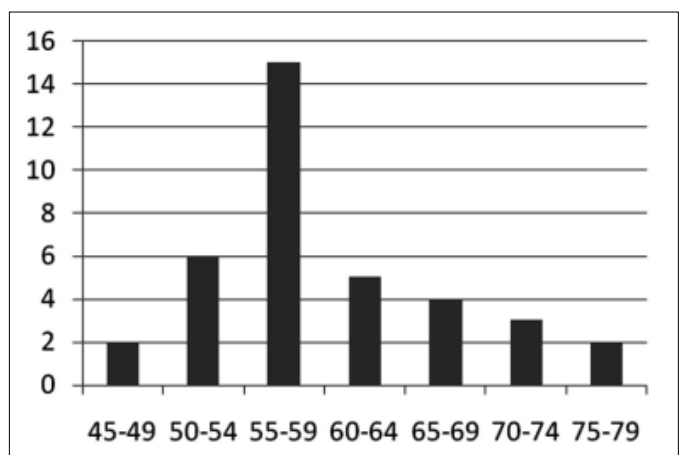

Figura 2 -Distribuição dos participantes por grupos de idade.

Contudo, é possível perceber que na execução de algumas de habilidades as pessoas com mais idade tiveram maiores dificuldades. A Tabela 3 apresenta a correlação (Pearson) entre a idade e a competência em lidar com as habilidades.

Tabela 3 - Correlação entre idade e habilidades.

\begin{tabular}{cccc}
\hline Habilidade & Momento da medição & Correlação (Pearson) & $p$ \\
\hline Lidar com mouse & Início do curso & 0,240 & 0,153 \\
& Final do curso & $0,356^{\star}$ & 0,030 \\
Orientação na tela & Início do curso & 0,322 & 0,052 \\
& Final do curso & 0,216 & 0,199 \\
Abrir o programa & Início do curso & 0,217 & 0,198 \\
Fechar o programa & Final do curso & 0,280 & 0,093 \\
& Início do curso & 0,290 & 0,082 \\
Digitar & Final do curso & $0,522^{\star *}$ & 0,001 \\
& Início do curso & 0,270 & 0,128 \\
Relação tela e mouse & Final do curso & 0,144 & 0,395 \\
& Início do curso & 0,322 & 0,052 \\
\hline
\end{tabular}

** Correlação significativa para um nível de significância de 0,01.

* Correlação significativa para um nível de significância de 0,05. 
A análise dos dados demonstra que no início do curso existe uma correlação entre as habilidades e a idade muito fraca, que não alcança em nenhuma um nível de significância. Porém, no final do curso pode-se perceber que existem três habilidades que apontam para diferenças significativas em relação à idade: uso do mouse, relação tela-mouse e fechar o programa. Isso significa que as pessoas mais velhas não conseguiram avançar no desenvolvimento dessas habilidades de mesma forma que as mais jovens.

Essa diferença entre os participantes mais jovens e mais velhos pode ter diferentes causas. Na literatura sobre aprendizagem de pessoas idosas são apontadas três possíveis causas: mudanças motivacionais, mudanças sensoriais e alterações físicas, principalmente em relação à velocidade das operações cognitivas. (LEHR, 2000).

Os mecanismos responsáveis por essa lentificação estariam relacionados à diminuição da velocidade de transmissão sináptica ou perda de informação em cada transmissão. (GUERREIRO; CALDAS, 2001). O uso do mouse, porém, não exige somente operações cognitivas, mas também motoras e sensoriais. Nesse sentido, a menor destreza dos participantes mais idosos ao usar o mouse pode estar associada ao fato de que a aprendizagem desta tarefa requer, além das operações cognitivas, habilidades sensoriais e motoras, e com o avançar da idade o indivíduo, gradativamente, vai perdendo a eficácia de todas as suas funções nervosas (IZQUIERDO, 2002), especialmente das sensoriais e motoras e do ritmo de processamento.
Os participantes mais velhos, provavelmente, não avançaram da mesma forma que os mais jovens no desempenho de algumas tarefas, por necessitarem de mais tempo para alcançar o mesmo nível de aprimoramento. Esse fato se relaciona com a orientação da atenção na aprendizagem e está associado à capacidade do sujeito de selecionar algumas informações e inibir aquelas que são irrelevantes para o desempenho de determinada tarefa. Luria (1991) destaca dois grupos de fatores - internos e externos - que asseguram o caráter seletivo dos processos psíquicos. Os processos internos estão relacionados com o próprio sujeito. A este grupo pertence a influência exercida pelas necessidades, os interesses e os objetivos do sujeito. Assim, a força desses fatores é muito importante nos processos de atenção e aprendizagem.

Os participantes do curso tinham clareza sobre a necessidade da aprendizagem de informática para o seu cotidiano, pois buscavam o curso com objetivos definidos, para o uso do computador nas suas vidas cotidianas. Mesmo assim, é possível, que haja uma diferença entre os participantes mais jovens, que geralmente procuraram o curso por questões profissionais, e os mais velhos, que o procuraram por interesses voltados ao mundo privado, como comunicação com os filhos e netos e para se sentirem inseridos no mundo digital atual. Contudo, geralmente as necessidades profissionais tendem a exercer uma pressão motivacional mais forte.

O segundo grupo de fatores determinantes do sentido da atenção, segundo 
Luria (1991), é constituído por fatores externos, que são os estímulos exteriormente perceptíveis pelo sujeito: como a intensidade (grandeza, cor) e a novidade do estímulo. Dessa forma, parecem residir neste segundo grupo as maiores dificuldades dos sujeitos mais velhos. Os déficits visuais - próprios da idade - e as condições específicas do equipamento (tamanho da tela e ícones) podem prolongar o tempo necessário para certas habilidades serem automatizadas.

Outro fator que pode interferir no direcionamento da atenção é o fato de que um estímulo novo começa imediatamente a atrair para si atenção. Nesse sentido, destacamos que as pessoas mais velhas têm uma tendência a serem mais dispersas (BRUCKI, 2004), e qualquer novo estímulo pode interferir no desempenho das tarefas. Esse fato é explicado, conforme Gurreiro e Caldas (2001), em razão da falta de inibição no processamento da memória operacional.

A memória operacional, também conceituada como memória de trabalho (IZQUIERDO, 2002), é curta, dura desde alguns segundos até no máximo 1-3 minutos. O seu papel principal não é o de formar memórias, mas, sim, analisar informações que chegam constantemente ao cérebro. Quando estamos evocando determinada experiência ou conhecimento, por exemplo, procurando abrir um programa no computador, ativa-se a memória de trabalho para verificar se esta memória já foi formada e consolidada. Além desta função, de determinar se a informação é nova ou não, a memória de trabalho determina se ela é útil ou não. Por isso, as possibilidades de ocor- rer aprendizagem numa situação estão determinadas pela memória de trabalho e suas conexões com os demais sistemas mnêmicos. (IZQUIERDO, 2002).

Raciocínio e compreensão são tarefas que tipicamente dependem da memória operacional, pois esta não se restringe ao armazenamento temporário de informações, mas refere-se à integração temporal e à manipulação de vários tipos de informação relevantes para a ação imediata. (BUENO; OLIVEIRA, 2004). Assim, a redução da capacidade funcional da memória operacional é vivenciada como dificuldade de concentração. Para Sé et al. (2004) os idosos apresentam dificuldade de realizar tarefas simultâneas quando as informações não são armazenadas e processadas de maneira eficaz, e as perdas sensoriais podem ser responsáveis por certo declínio da memória operacional.

Essa questão pode explicar o fato do menor desempenho dos mais velhos na habilidade de relacionar a tela com o mouse. Ao realizar esse exercício os sujeitos necessitam coordenar mais de uma atividade. Conforme Taussik e Wagner (2006), estudos têm evidenciado que as dificuldades dos idosos estão em situações em que a memória precisa interagir com a atenção e com uma organização de ações. Esses processos se tornam ainda mais difíceis quando se trata de tarefas e elementos totalmente novos, pois não encontram estruturas cognitivas adequadas para operá-las. Como pessoas idosas, nas suas biografias, geralmente, tiveram menos chances de terem conhecido e usado computadores ou equipamentos digitais, precisam 
elaborar estruturas cognitivas novas, $o$ que demora mais tempo do que somente integrar informações em estruturas já existentes.

Quanto à habilidade de fechar um programa no computador, os participantes mais idosos também apresentaram um desempenho inferior ao dos mais jovens. O sujeito, no momento de executar essa tarefa, já realizou muitas outras atividades no computador. Dessa maneira, esse dado pode indicar um cansaço maior desses sujeitos - provenientes da ação de selecionar informações relevantes e inibir estímulos desnecessários para sua ação - já que o esforço despendido pela pessoa idosa é maior por se tratar, comumente, de aspectos novos e desconhecidos.

Finalmente, podemos inferir que os resultados da avaliação das habilidades realizadas neste estudo se relacionam com declínio das funções executivas, que pode ocorrer de forma mais acentuada a partir dos sessenta anos. Banhato e Nascimento (2007) destacam que não se pode esquecer que existem enormes diferenças intra e interindividuais. Além disso, é importante lembrar que a utilização constante das funções executivas e treinamentos específicos pode retardar ou até inverter possíveis perdas. (LEHR, 2000). Esses processos cognitivos

\footnotetext{
envolvem a focalização da atenção em informações relevantes, inibição de processos e informações irrelevantes ou concorrentes, programação de processos para tarefas complexas que necessitam alternância entre elas, planejamento de seqüências de subtarefas e monitoramento do desempenho para a execução de um comportamento dirigido a objetivos. (KRISTENSEN, 2006, p. 97).
}

Conforme Dos Santos (2004), essas operações permitem ao indivíduo interagir intencionalmente no mundo, fazer planos de ação, e visam ao controle e à regulação do processamento da informação.

Também é interessante observar o desenvolvimento das habilidades nas quais a influência da idade parece diminuir durante o curso. Trata-se da orientação na tela do computador e da digitação. A análise da primeira aprendizagem está associada à orientação visoespacial. A análise sugere que a informação sobre localização dos ícones que constituem a tela do computador foi sendo consolidada e que esse processo não sofreu influência da idade.

As dificuldades ligadas à idade em relação à memória aumentam com a complexidade da tarefa (GUERREIRO; CALDAS, 2001), indicando, assim, o declínio relacionado às funções executivas. No caso da orientação na tela, não há necessidade de o sujeito coordenar ações. Portanto, por ser tratar de uma tarefa mais simples, o idoso pode ter codificado, armazenado e resgatado a informação sem maiores dificuldades.

Em relação à digitação, entendemos que as pessoas mais velhas, que já possuíam experiência em digitar em máquinas de escrever, reativaram seus conhecimentos. Digitar é uma habilidade adquirida por meio de processos de formação de memórias procedurais ou "hábitos". Conforme Izquierdo (2002), essas memórias provém da aquisição de habilidades sensoriais e motoras e são obtidas de maneira implícita e mais ou menos automática, sendo difícil para o 
sujeito descrever os passos da aquisição, por exemplo, da capacidade de digitar. A memória procedural não mostra prejuízos com o avançar da idade, sendo escassos os estudos sobre este tipo de memória pelo fato de que raras vezes essas apresentam falhas. (Idem, 2002). Já os sujeitos mais jovens, que não aprenderam a digitar, precisariam de mais tempo e treino para avançar nessa habilidade.

\section{Conclusões e perspectivas}

Embora nosso estudo apresente limitações em razão do número pequeno de sujeitos, foi possível produzir discussões pertinentes acerca dos processos de aprendizagem de adultos maduros e idosos. Destacamos como elementos facilitadores do aumento de desempenho dos sujeitos ao longo do curso algumas características específicas do grupo pesquisado, como as motivações e o uso do computador em casa. Ter perspectiva de como usar o computador, buscar as informações necessárias para realizar ações de que necessita ou que deseja e treinar após as aulas foram elementos considerados decisivos para o progresso na aprendizagem das habilidades avaliadas. Também destacamos como relevante $o$ alto e relativamente homogêneo grau de escolaridade dos participantes do curso, reforçando a ideia de que a educação formal, em geral, facilita o desempenho intelectual.

O menor desempenho dos participantes idosos em algumas habilidades demonstrou dificuldades relacionadas às funções executivas. Esses dados corroboram com os resultados de um estudo transversal brasileiro que avaliou funções executivas de idosos. Esta pesquisa demonstrou que a idade exerceu influência significativa nos desempenhos da atenção, da memória de trabalho e da organização visoespacial, sendo a velocidade de processamento de informação a mais prejudicada. (BANHATO; NASCIMENTO, 2007). Apesar da importância da velocidade para lidar com um meio novo como o computador, existem, obviamente, outros fatores que podem explicar o menor desempenho das pessoas mais idosas, como, por exemplo, questões motivacionais, circunstâncias da vida ou até restrições sensoriais. (MORAGAS, 1997; LEHR, 2000).

Esses resultados confirmam as observações acerca das atitudes dos sujeitos realizadas ao longo do curso. Diversas vezes os idosos faziam muitas perguntas sobre estímulos diversos e não relacionados às explicações da professora. Também era comum vê-los detidos em outras tarefas que não aquelas solicitadas no momento da aula. Isso poderia ser entendido como dificuldade de concentração, mas, ao mesmo tempo, esta postura se relaciona ao deslumbramento com o desconhecido - a informática. O encantamento com a possibilidade de "entrar" no mundo digital, por um lado, os motiva, mas, por outro, pode dispersá-los. A fala abaixo demonstra como um dos participantes relata sentirse à frente do computador:

Deslumbrado. Eu me sinto assim, como se eu tivesse no globo terrestre e eu tivesse no meio. (Ricardo, 71 anos)

Neste artigo utilizamos uma perspectiva psicológica cognitiva como referência para analisar os dados obtidos durante um curso de computação por 
meio de um instrumento de observação. Como mencionado, trata-se somente de uma abordagem possível para explicar diferenças de desempenho de pessoas durante um processo de aprendizagem.

Entendemos que existem diferentes fatores que facilitam ou dificultam a aprendizagem de lidar com o computador e que determinam o uso posterior do aprendido, quais sejam: escolaridade; idade; características pessoais, como curiosidade, medo, insegurança; funções cognitivas, como atenção e memória; grau de perspectiva concreta do uso do computador e acesso ao computador. Nesse sentido, a proposta educativa e a avaliação dos processos de aprendizagem digital devem levar em consideração todos os aspectos que integram os processos de aprendizagem de adultos e idosos.

\section{Learning in courses of digital inclusion} for adults and older

\section{Abstract}

The digital inclusion in Brazil is going on, but not in the same way for all ages: most of the adult and elderly don't know how to use a computer and there are little efforts to include this specific group in the digital community. In order to understand better this challenge, the present study analyzes the learning process of people with 45 years and more during computer courses for beginners, designed for this age group, by using a special instrument to register the performance in a group of abilities. The data show different rhythms of learning, and a certain influence of age in some abilities. The results are discussed from the point of view of psychological learning theories.

Key words: Digital inclusion. Learning. Aging.

\section{Referências}

BANHATO, E. F.; NASCIMENTO, E. Função executiva em idosos: um estudo utilizando subtestes da Escala WAIS-III. PsicoUSF, Itatiba, v. 12, n. 1, p. 1-13, jun. 2007.

BUENO, O. F.; OLIVEIRA, M. G. M. Memória e amnésia. In: ANDRADE, V. M.; SANTOS, F. H.; BUENO, O. F. A. Neuropsicologia hoje. São Paulo: Artes Médicas, 2004. p. 135-163.

BRUCKI, S. M. D. Envelhecimento e memória. In: ANDRADE, V. M.; SANTOS, F. H.; BUENO, O. F. A. Neuropsicologia hoje. São Paulo: Artes Médicas, 2004. p. 389-402.

DOH, M. Erobern silver-surfer das netz? Stiftung digitale chance. Disponível em: <www. stepping-stones.de/content/stories/index.cfm/ aus.10/key.905/secid.16/secid2.49>. Acesso em: 6 nov. 2007.

DOS SANTOS, F. H. Funções executivas. In: ANDRADE, V. M.; SANTOS, F. H.; BUENO, O. F. A. Neuropsicologia hoje. São Paulo: Artes Médicas, 2004. p. 125-134.

DOLL, J. Pedagogia social e a realidade brasileira. In: ENCONTRO IBERO-AMERICANO: A INTERVENÇÃO EDUCATIVA NA VELHICE DESDE A PERSPECTIVA DE UMA PEDAGOGIA SOCIAL, I, 2002, Caxias do Sul. Anais... Caxias do Sul: Universidade de Caxias do Sul, 2002. p. 48-53.

Educação, cultura e lazer: perspectivas de velhice bem-sucedida. In: NERI, A. L. (Org.). Idosos no Brasil: vivências, desafios e expectativas na terceira idade. São Paulo: Fundação Perseu Abramo; Edições Sesc/SP, 2007. p. 109-124.

GUERREIRO, T.; CALDAS, C. P. Memória e demência: (re)conhecimento e cuidado. Rio de Janeiro: Uerj; Unati, 2001.

IZQUIERDO, I. Memória. Porto Alegre: Artmed, 2002.

KACHAR, V. Terceira idade e informática: aprender revelando potencialidades. São Paulo: Cortez, 2003. 
KRISTENSEN, C. H. Funções executivas e envelhecimento. In: PARENTE, M. A. M. P. Cognição e envelhecimento. Porto Alegre: Artmed, 2006. p. 97-111.

LEHR, U. Psychologie des Alterns. 9. ed. Wiebelsheim: Quelle und Meyer, 2000.

LURIA, A. R. Curso de psicologia geral: atenção e memória. 2. ed. Rio de Janeiro: Civilização Brasileira, 1991.

MORAGAS, Ricardo. Gerontologia social: envelhecimento e qualidade de vida. São Paulo: Paulinas, 1997.

NABAS, T. R.; XAVIER, G. F. Neurobiologia da atenção visual. In: ANDRADE, V. M.; SANTOS, F. H.; BUENO, O. F. A. Neuropsicologia hoje. São Paulo: Artes Médicas, 2004. p. 101-124.

SÉ, E. V. G.; QUEIROZ, N. C.; YASSUDA, M. S. O Envelhecimento do cérebro e a memória. In: NERI, A. L.; YASSUDA, M. (Org.). Velhice bem-sucedida: aspectos afetivos e cognitivos. Campinas: Papirus, 2004.

SELWYN, N. et al. Older adults' use of information and communications technology in everyday life. Aging and Society, v. 23, n. 5, p. 561-582, 2003.

TAUSSIK, I.; WAGNER, G. Memória explícita e envelhecimento. In: PARENTE, M. A. M. P. Cognição e envelhecimento. Porto Alegre: Artmed, 2006. p. 67-84.

US DEPARTMENT OF COMMERCE. Falling through the NET: toward digital inclusion - A report on Americans' Access to Technology Tools. Washington, 2000. 